Cahiers $d u$ MONDE RUSSE

\section{Cahiers du monde russe}

Russie - Empire russe - Union soviétique et États indépendants

$50 / 2-3 \mid 2009$

L'Europe orientale, 1650-1730. Crises, conflits et renouveau

\title{
Correspondance sur la religion entre un Russe et un Anglais (1686-1687)
}

Correspondence between an Englishman and a Russian regarding religion (1686-1687)

\section{Petr Sergeevič Stefanovič}

\section{OpenEdition \\ Journals}

Édition électronique

URL : https://journals.openedition.org/monderusse/9723

DOI : 10.4000/monderusse. 9723

ISSN : $1777-5388$

Éditeur

Éditions de l'EHESS

Édition imprimée

Date de publication : 15 septembre 2009

Pagination : 453-463

ISBN : 978-2-7132-2260-3

ISSN : $1252-6576$

Référence électronique

Petr Sergeevič Stefanovič, "Correspondance sur la religion entre un Russe et un Anglais

(1686-1687) », Cahiers du monde russe [En ligne], 50/2-3 | 2009, mis en ligne le 13 octobre 2012,

consulté le 04 septembre 2022. URL : http://journals.openedition.org/monderusse/9723 ; DOI :

https://doi.org/10.4000/monderusse. 9723 


\section{CORRESPONDANCE SUR LA RELIGION ENTRE UN RUSSE ET UN ANGLAIS (1686-1687)}

Le département des manuscrits de la British Library à Londres conserve un recueil factice polyglotte qui comprend, outre différents documents et des œuvres littéraires, plusieurs cahiers en cyrillique ${ }^{1}$. La partie cyrillique du recueil réunit, de même, des matériaux hétérogènes en apparence, mais qui, à l'examen, remontent à une source commune ${ }^{2}$. Ils proviennent tous, en effet, des archives d'un marchand anglais, qui a vécu à Pskov dans les années 1680, y faisait du commerce et y apprenait le russe. L'essentiel est constitué d'une correspondance entre cet Anglais et un citadin de Pskov, dont l'activité principale semblait avoir été l'enseignement du russe aux étrangers. Dans ses lettres, l'habitant de Pskov appelle l'Anglais «Roman Vilimovič » et ce dernier l'appelle «Pëtr Ignat'evič » (ci-après : R.V. et P.I.). Les lettres sont datées de juin à août 1686 et de mars à avril 1687, en tout treize lettres de P.I. et huit de R.V. L'activité épistolaire faisait partie de l'enseignement du russe, c'est pourquoi toutes les lettres en russe portent la trace de corrections de P. I. ${ }^{3}$

1. A Catalogue of the Harleian Manuscripts in the British Museum, vol. III, Londres, 1808, p. 359-360.

2. Pour une description des cahiers en cyrillique du manuscrit, voir V. Du Feu et W.F. Ryan, éds., A Union Catalogue of Cyrillic Manuscripts in British and Irish Collections, compiled by Ralph Cleminson, Londres : School of Slavonic and East European Studies, 1988, p. 163-166.

3. Pour plus de détails, voir Petr S. Stefanovič, « Rukopis` Britanskoj biblioteki s perepiskoj Pskoviča i anglijskogo kupca 80-h godov XvII veka » [« Un manuscrit de la British Library, contenant la correspondance d'un habitant de Pskov avec un marchand anglais des années 1680 »], Pskov v rossijskoj i evropejskoj istorii : Meždunarodnaja naučnaja konferencija, [Pskov dans l'histoire de la Russie et de l'Europe : Conférence scientifique internationale], t. 1, Pskov, 2003. La correspondance a été publiée avec le soutien du RGNF (Rossijskij gumanitarnyj naučnyj fond - Fonds russe pour les sciences humaines), (projet $\left.\mathrm{N}^{\circ} 05-01-0107 \mathrm{ba}\right)$ : Boris Nikolaevič Morozov, Petr Sergeevič Stefanovič, éds., Roman Vilimovič v gostjah u Petra Ignat'eviča : pskovskij arhiv anglijskogo kupca 1680-h godov [Roman Vilimovič invité de Petr Ignat'evič : archives de Pskov d'un marchand anglais des années 1680], M. : Indrik, 2009. 
Ce dernier non seulement a enseigné le russe à R.V., mais il lui a également loué quelque temps un logement attenant à sa maison. Ils échangeaient des livres, et leur correspondance ne laisse aucun doute quant aux relations amicales qui s'étaient instaurées entre le maître et l'élève. Leur rencontre à Pskov n'était certes pas le fruit du hasard : cette ville était en effet l'ultime frontière de la Moscovie vers l'Ouest. C'est par là que passaient les échanges commerciaux avec les pays occidentaux et les étrangers y étaient présents depuis toujours. Les sources écrites font remonter à la fin du XIII ${ }^{\mathrm{e}}$ siècle au moins la tradition de l'enseignement du russe aux étrangers, tout d'abord à Novgorod, puis, plus tard, à Pskov. Les élèves étaient le plus souvent de jeunes gens de familles marchandes envoyés là par leurs aînés. Tönnies Fenne, qui rédigea en 1607 le fameux dictionnaire et manuel de conversation russo-allemand $^{4}$, en donne un parfait exemple. Certes, la politique d'isolation et de ségrégation des étrangers menée par le gouvernement russe au $\mathrm{XVII}^{\mathrm{e}}$ siècle n'était pas sans imprimer sa marque ${ }^{5}$, comme en témoignent certaines manifestations de xénophobie lors de la révolte de Pskov en $1650^{6}$. Néanmoins, la ville restait dans l'ensemble un centre important de contacts internationaux. De plus, Pskov était l'un des foyers de la culture urbaine, en plein essor au XVII ${ }^{\mathrm{e}}$ siècle, et ce sont précisément les conditions socio-culturelles du milieu des marchands qui ont rendu possible ce « dialogue des cultures » d'un type particulier.

Malheureusement, nous ne disposons pas d'autres sources qui nous permettraient de mieux identifier les correspondants. À en juger par les réalités évoquées dans les lettres et autres documents de ces « archives », P.I. faisait partie de la population aisée de la ville et quelques indices autorisent à supposer qu'il appartenait à une lignée de marchands de Pskov, les Koljagin. Le plus probant est l'inscription commémorative qui énumère les fondateurs de l'église de la Dormition d'OutreVelikaja, face au Pont flottant ${ }^{7}$. Gravée sur une plaque de bronze, puis emmurée

4. L.L. Hammerich, Roman Jakobson et alii, éds., Tönnies Fenne's Low German Manual of Spoken Russian, Pskov 1607, 2 vol., Copenhague : Det Kongelige Danske Videnskabernes Selskab, 1960-1970. Voir également Pierre Jeannin, « Der Lübecker Tönnies Fonne - ein Pionier der Slawistik », Hansische Geschichtsblätter, 91, 1973, p. 50-53 ; du même auteur, «Entre Russie et Occident au début du XVII siècle : le contexte historique d'un grand document linguistique », Études européennes. Mélanges offerts à Victor L. Tapié, P. : Publications de la Sorbonne (Série Études, 6), 1973, p. 503-524, repr. dans P. Jeannin, Marchands du Nord : espaces et trafics à l'époque moderne, P. : PEN, 1996, p. 165-182 ; A.L. Horoškevič, « Byt i kul'tura russkogo goroda po slovarju Tonni Fenne 1607 g. » [ « La vie quotidienne et la culture d'une ville russe d'après le dictionnaire de Tönnies Fenne de 1607 »], in V.A. Aleksandrov, éd. et al., Novoe o prošlom našej strany [Du nouveau sur le passé de notre pays], M. : Nauka, 1967, p. 200-217.

5. Pour plus de détails sur les pressions que subissaient les étrangers en Russie aux XVI ${ }^{\mathrm{e}}-\mathrm{XVII}{ }^{\mathrm{e}}$ siècles, voir Marshall T. Poe, « A People Born To Slavery », Russia in Early Modern European Ethnography, 1476-1748, Ithaca et Londres : Cornell University Press, 2000, p. 41-49, 83-90.

6. Vladimir Anatol'evič Arakčeev, Srednevekovyj Pskov : vlast', obščestvo, povsednevnaja žizn' $V X V$-XVII vekah [Pskov médiévale : pouvoir, société, vie quotidienne aux $X V^{e}-X V I I^{e}$ siècles], Pskov : Pskovskaja oblastnaja tipografija, 2004, p. 214.

7. «[...] s Paromenija v Zavelič'e » : le Zaveliče était un quartier situé de l'autre côté de la rivière Velikaja (qui traverse Pskov) par rapport à la citadelle ou « kremlin ». Venant du centreville, on y accédait par le Paromen'e, ou pont flottant. Cf. Inga Konstantinovna Labutina, 
lors de la reconstruction de l'église en 1693, elle mentionnait parmi les membres de cette famille un certain Pëtr, fils d'Ignatij ${ }^{8}$.

Nous avons choisi de nous concentrer ici sur les lettres de P.I. qui nous paraissent plus intéressantes que les lettres de R.V. à bien des égards : plus nombreuses, plus étoffées, elles sont également plus variées. Même en parcourant superficiellement ces lettres, il est frappant de constater que leur auteur met toujours l'accent sur l'aspect religieux des thèmes abordés, qu'il s'agisse de considérations d'ordre général ou de problèmes particuliers. À plusieurs reprises, les correspondants abordent directement les questions touchant la « foi chrétienne ». Les opinions de l'habitant de Pskov sur la foi, sa description des rituels orthodoxes, les jugements qu'il porte sur d'autres sujets, pénétrés qu'ils sont d'une vision religieuse du monde, permettent d'en tirer quelques conclusions sur ses sentiments religieux. Nous exposerons dans un premier temps ses opinions et ses jugements, puis nous comparerons nos observations à d'autres cas de « dialogue religieux » à l'époque précédant les réformes de Pierre le Grand.

Dans sa forme (les formules stéréotypées d'adresse et de vœux, les formules de politesse, etc.) la correspondance est tout à fait conforme à la tradition russe, ce qui n'est pas étonnant, puisque l'acquisition des pratiques épistolaires faisait partie de l'enseignement. Que dans ses rapports épistolaires avec un étranger, un habitant de Pskov ait recours aux mêmes formules que celles qu'il aurait utilisées avec un Russe, n'en reste pas moins un fait révélateur. En effet, quelque formels que puissent être ces énoncés, ils étaient cependant porteurs d'un contenu réel, dont P.I. n'hésite pas à faire bénéficier R.V. Il l'appelle son « élève respectueux » (smirennyj učenik), et même son « bienfaiteur » (blagodetel $)$, « homme vertueux » (dobrodej), allant jusqu'à se déclarer coupable devant lui, « comme un esclave devant son maître » $\left(1.302 \mathrm{v}^{\circ}\right)^{9}$. Mais le plus souvent l'habitant de Pskov s'adresse à son élève anglais comme à son « ami », son «bon ami » [drug, prijatel $)$, une fois même comme à son « ami bienveillant et de bon conseil » (milostivoj moj i sovetnyj drug) (1. 333). Il nous semble que ces formules ne sont pas fortuites, et cela pour deux raisons.

Tout d'abord, R.V. payait à P.I. ses leçons et le logement, il lui rendait également quelques services, en lui prêtant de l'argent ou en lui faisant des cadeaux. P.I. lui en était reconnaissant et il lui accordait une confiance toute particulière, d'autant que R.V. était visiblement un bon élève. Les documents de ces « archives » évoquent en outre d'autres étrangers étudiant chez P.I. et, en tant que précepteur professionnel, l'habitant de Pskov se devait de manifester de l'intérêt pour ses « amis » étrangers. En second lieu, le terme d' «ami » (drug) correspond à l'idée que

Istoričeskaja topografija Pskova V XIV-XV VV. [Topographie historique de Pskov, XIV $\left.{ }^{e}-X V^{e} s s.\right]$, M. : Nauka, 1985, p. 46-47, 123-124, 224 (NdT).

8. Taisija Viktorovna Kruglova, Elena Aleksandrovna Jakovleva, « K istorii Uspenskogo hrama v Pskove » [ « Pour contribuer à une histoire de l'église de la Dormition de Pskov»], in V.V. Sedov, éd., Drevnosti Pskova : Arheologija, istorija, arhitektura. K jubileju I.K. Labutinoj, [Les antiquités de Pskov: archéologie, histoire, architecture. À l'occasion de l'anniversaire de Inga Konstantinovna Labutina], Pskov, 1999, p. 102.

9. Les références des citations renvoient aux folios du manuscrit. 
P.I. prêche avec insistance dans ses lettres, à savoir que tous les chrétiens doivent s'aimer les uns les autres comme des frères, être des amis fidèles et attentionnés.

Dans l'une de ses lettres, P.I. énonce cette pensée de la façon suivante. Selon lui, son amitié avec R.V. est fondée sur les commandements divins :

Et Dieu a écrit ceci : chaque homme doit éprouver de l'amour ou de l'amitié pour autrui. L'apôtre Jean a écrit : qui aime Dieu et déteste ou n'aime point son frère, celui-là vit dans le mensonge et dans l'injustice. Comment l'homme peut-il aimer Dieu ? Il ne voit pas Dieu. Il convient dans un premier temps d'aimer tout homme de bien, puis il faut aimer Dieu de même. Dieu enjoint à l'homme de n'être point orgueilleux, ni arrogant, et de ne point éprouver de colère envers ses amis ou envers toute autre personne. Il est écrit dans nos livres que l'amour rachète de nombreux péchés. Alors, que Dieu nous accorde à nous aussi amour et bonne amitié [...]. (1.324 $\left.\mathrm{v}^{\circ}-325\right)$

Cette pensée peut sembler banale, mais, à l'époque en question, son évocation dans la correspondance entre un Russe et un étranger est tout à fait inattendue et sans doute unique. La façon dont l'Anglais adhère à cette exhortation est non moins surprenante que l'« appel œcuménique » du Russe. Dans sa réponse, R.V. développe cette idée :

quant à ce que tu m'écris que Dieu n'aime point celui qui n'aime point son propre frère, je pense que cela est vrai, et que nous, chrétiens, sommes nés sur la Terre d'un même Père [...]. (1.326)

Fort de cette compréhension mutuelle, P.I. applique directement sa pensée à ses relations de maître à élève. Dans l'une des lettres suivantes, P.I. demande à R.V. :

[as-tu] toujours avec tous tes amis des relations d'amitié et de bonne entente, et [n'y a-t-il] point eu avec l'un d'entre eux une querelle ou une mésentente [?] Je suis heureux d'entendre que tu as toujours conservé avec tes amis des relations d'amitié et de bonne entente, comme il est écrit dans les Écritures Saintes.

Pour décrire le comportement d'un chrétien authentique, P.I. ne craint pas de se donner en exemple :

Et Dieu m'a accordé d'être en vie à ce jour, poursuit le Russe, et de vivre dans l'amitié et la bonne entente avec tous mes amis et tous les gens de bien. Et s'il m'arrive quelquefois d'échanger avec certains d'entre eux des propos malsonnants ou de leur chercher querelle, nous nous pardonnons aussitôt mutuellement et continuons de vivre en bonne entente, n'en déplaise au diable. De même, puisque tu mènes une vie tranquille et paisible, dans la bonne entente avec tes amis, cela est très bien et Dieu l'a voulu ainsi, et tous les gens de bien te loueront et te respecteront, de même que moi, je fais vraiment partout ton éloge pour ta modestie et toutes tes vertus. $\left(1.328 \mathrm{v}^{\circ}-329 \mathrm{v}^{\circ}\right)$ 
Dans ses lettres à son correspondant anglais, le «professeur » de Pskov met donc l'accent sur la morale sociale préconisée par la doctrine chrétienne. Vivre avec tous les « gens de bien » dans la paix et la bonne entente, voilà le credo de P.I. Comme synonyme de «bon, bien », il emploie « modeste, humble » (smirennyj), c'est-à-dire calme, ni impulsif, ni intrigant, ni querelleur. Et l' « humilité » (smirenie) devient la caractéristique essentielle du comportement de l' « homme de bien ». Selon toute vraisemblance, tous les chrétiens sont des « gens de bien » en puissance.

P.I. exprime à diverses reprises sa reconnaissance envers son élève, autant pour son comportement amical que pour les services qu'il lui a rendus. Il le remercie ainsi, entre autres, de savoir tenir sa langue et du tact dont il fait preuve. R.V. remercie en retour P.I. pour tout le « bien » (dobrodeteli) qu'il a retiré de son séjour chez P.I., et le Russe de lui répondre :

[tu] me gratifies de ta bienveillance, mais je ne sais pourquoi, car tu n'as pu trouver que peu de bien sous mon toit ou de ma part. Je prie au décuple pour ta santé, pour ton humilité et ta discrétion, tu as été témoin de bien vilaines choses chez moi, mais tu ne l'as ébruité nulle part, et ne l'as raconté à personne. Un tel bienfait est inoubliable. Que Dieu t'accorde pour cela tout le bien que tu attends de lui, notre Créateur. Si d'aventure un autre élève s'était trouvé chez moi et avait entendu ce qui s'y est produit lorsque tu étais là, il y a longtemps que les gens le sauraient et qu'ils riraient de moi. $\left(1.333-\mathrm{v}^{\circ}\right)$

En « homme de bien », P.I. fait preuve d'une large hospitalité et prend grand soin de son élève et locataire. Lorsque l'élève anglais ne loge plus chez P.I., ils se rendent mutuellement visite. L'une des lettres de P.I., justement, est une invitation, dans laquelle il souhaite à son élève « de se bien porter durant de longues années, avec ses parents et ses amis », et l'invite chez lui «pour la fête de la résurrection du Christ »: «Fais-moi cette grâce, viens chez moi à l'occasion de la fête boire de la bière à la fortune du pot ». Après une fin de carême probablement festive, P.I. plaint son ami indisposé et lui donne un « conseil médical »: «j'ai entendu dire que tu as la gueule de bois aujourd'hui, prie Dieu qu'Il te fasse grâce, et tu seras comme avant $\gg\left(1.337,338 \mathrm{v}^{\circ}\right)$.

Les fondements religieux de la morale sociale de P.I. ne font aucun doute. De même, le fait que le maître demande plusieurs fois pardon à son élève de sa paresse, « au nom du Seigneur ou pour l'amour de Dieu », fait supposer que sa morale économique avait elle aussi des racines religieuses, même si ces dernières ne sont pas exprimées de façon explicite dans la correspondance. De toutes façons, l'une et l'autre se fondent, chez P.I., en une seule et même éthique. Dans sa première lettre, P.I. s'exprime sans ambiguïté sur ce point :

[...] Et aujourd'hui, je pense à part moi, et j'ai très honte vis-à-vis des gens de bien russes et étrangers, car je me suis souvent enivré, et j'ai toujours beaucoup bu, j'ai été oisif, je ne t'ai rien écrit et, à cause de ma paresse, tu as passé beaucoup de temps sans recevoir un enseignement. Je te demande pardon pour 
tout cela, pardonne-moi, s’il te plaît. Désormais, j'écrirai plus souvent à Ta Seigneurie. (1.303)

P.I. ne se contentait pas de demander pardon de sa paresse, il rappelait aussi à R.V. de ne pas oublier son apprentissage et d'écrire plus souvent, pour exercer son russe.

L'ivrognerie, qu'il évoque à plusieurs reprises, était considérée par P.I. comme un péché, dans la mesure où elle éloignait du travail et de la fréquentation de l'église. Dans une lettre qui décrit la façon de fêter Pâques en Russie, P.I. donne l'exemple d'une mauvaise célébration ( « charnelle »), par opposition à une célébration authentique («spirituelle») puis il poursuit avec une mise en garde au sujet de la fin du carême :

Il nous est fermement recommandé de fêter la semaine sainte selon l'esprit, et non selon la chair. Qu'est-ce qui est selon l'esprit ou selon la chair, je vais te le dire. Agir selon l'esprit, c'est prier Dieu, aller à l'église, boire et manger modérément, éviter l'indigestion et l'ivresse. Agir selon la chair, c'est manger et boire excessivement, s'enivrer, dormir ou pécher d'autre façon, et ne pas aller à l'église. C'est encore obéir à la chair, c'est-à-dire pécher, que de forniquer en état d'ivresse, de chanter des chansons grossières et se comporter de façon indécente, en oubliant de prier Dieu. $\left(1.337 \mathrm{v}^{\circ}\right)$

Le sentiment religieux chez P.I. va donc bien au-delà d'une éthique sociale. Si P.I. insiste particulièrement sur ce dernier aspect, c'est à cause de la situation : il convenait de démontrer à l'étranger ses dispositions amicales et son hospitalité. La vie de l'église, et même, plus précisément, le rituel, avait en effet pour P.I. autant, voire plus d'importance que la morale. En témoignent la lettre citée ci-dessus, mais également l'une des deux œuvres littéraires dont on trouve une copie dans les archives du correspondant anglais : la « Parabole sur le fait qu'il convient de ne pas quitter l'église tant qu'on y chante ». Comme il ressort du « billet » de P.I. qui l'accompagne, il avait lui-même choisi et copié cette parabole à l'intention de R.V . (1. 302). Elle visait à démontrer combien il était important de fréquenter les offices religieux ${ }^{10}$. Nous pensons que ce choix n'est pas un hasard et reflète la conviction intime de P.I. lui-même.

Outre cette parabole, c'est très vraisemblablement P.I. qui recopia pour R.V toute une série de prières, d'exégèses et de sermons : la doxologie, le Notre Père, les Dix commandements, le Symbole de la foi avec gloses, quelques homélies, De la pénitence, Du baptême, etc. Ce choix obéit à une logique : P.I. souhaitait manifestement initier R.V. aux rudiments du dogme et du rituel orthodoxes. C'est pourquoi, dans la lettre sur Pâques citée précédemment, P.I. décrit encore à R.V . les particularités de l'office orthodoxe de Pâques (l'échange du baiser pascal, etc.).

10. Texte de la parabole dans D.S. Lihačev, L.A. Dmitriev, éds., Biblioteka literatury Drevnej Rusi [Bibliothèque de littérature russe ancienne], vol. 10, SPb. : Nauka, 2000, p. 104-106. Pour plus de détails, voir $S K K D R$, II, (2), p. 219-220. 
Enfin, on relève une allusion aux idées sociales et politiques de P.I. dans l'une des pièces de ce recueil orthodoxe, le commentaire du Notre Père, que P.I. a certainement recopié dans un traité, mais en y ajoutant ses propres considérations. Voici l'interprétation de «donne-nous notre pain quotidien » :

On appelle pain quotidien tout ce dont l'homme a besoin : d'abord manger et boire, puis vêtements et chaussures, une maison et du bien, une épouse modeste, des enfants dociles, une maisonnée paisible, un pouvoir pieux et sans arrogance, des sujets loyaux, la paix et la santé, la gentillesse et le respect de ses bons amis, des voisins à qui on peut se fier. $\left(1.282 \mathrm{v}^{\circ}\right)$.

C'est en somme une esquisse de la vie en société, telle que se la représentait notre habitant de Pskov en cette fin du XvII ${ }^{\mathrm{e}}$ siècle. De même que dans les lettres, nous retrouvons ici la maison, les bons amis et les voisins, mais s'y ajoutent « le pouvoir pieux » et les « loyaux sujets ». La base de cet édifice social, c'est-à-dire la valeur sur laquelle il repose, est l' « humilité » (smirenie), qui embrasse les concepts de douceur, de quiétude et de calme.

Une conclusion intéressante et d'une importance considérable s'impose à la lecture des lettres de P.I. : la vision du monde qu'expose l'habitant de Pskov se révèle sensiblement, et même radicalement différente de celle des Moscovites avant Pierre le Grand, telle que la décrivent d'ordinaire les historiens à partir des écrits d'Occidentaux de passage en Russie aux XVI ${ }^{\mathrm{e}}$ et XVII ${ }^{\mathrm{e}}$ siècles ${ }^{11}$. Dans les lettres de P.I., nous ne relevons ni la foi explicite dans les miracles, ni le refus de se raser la barbe, ni la méfiance à l'égard des étrangers. Dans les rapports entre P.I. et R.V., on n'observe ni préjugé, ni crainte de souillure : R.V. vit et prend ses repas chez P.I., fréquente sa famille, leur rend visite après son déménagement, notamment à Pâques pour le repas de fin de carême.

L'affirmation selon laquelle l'essence de l'orthodoxie réside dans la vénération des icônes et que, pour les Russes, le rituel est l'élément le plus important de la religion, est un lieu commun des écrits ouest-européens de l'époque (repris par les historiens contemporains). Or non seulement P.I. n'évoque jamais la vénération des icônes, mais à aucun moment il ne mentionne les saints (!). Il décrit les rites orthodoxes dans une optique qui n'est pas polémique, mais plutôt ethnographique. Dans le choix de prières, de sermons et d'exégèses qu'il effectue à l'intention de son élève anglais, P.I. insiste sur les principaux moyens de salut que sont les bonnes œuvres, l'amour, la pénitence et les sacrements de l'eucharistie et du baptême. Lorsque, dans sa lettre, il lui recommande de fréquenter l'église, il le fait de manière très générale : ce qui compte, c'est de vivre religieusement. Bien entendu, il faut tenir compte du fait que les relations entre P.I. et R.V. étaient purement privées et que leur rapports de maître à élève reposaient sur une « stratégie de communication » plutôt que sur

11. Voir, notamment, l'ouvrage classique de Vasilij Osipovič Ključevskij, Skazanija inostrancev o Moskovskom gosudarstve [Les récits d'étrangers sur la Moscovie], M. : Izd. Prometej im V.I. Lenina, 1991. 
une «stratégie de conflit ». Il n'en reste pas moins que leur recherche délibérée de points communs en matière religieuse contraste vivement avec les écrits des Occidentaux (catholiques et protestants) qui disent n'avoir presque jamais rencontré de Russes qui partagent leurs vues religieuses.

Pourtant on ne trouve aucune trace, dans les lettres de P.I., d'une quelconque européanisation ou d'une occidentalisation. Ni son style, ni ses jugements n'ont rien d'extraordinaire pour l'époque. Un élément essentiel de sa pensée religieuse s'inscrit même tout à fait dans le courant général de la dévotion russe du XVII siècle. Les historiens relèvent en effet que l'orthodoxie qui, jusqu'alors, avait privilégié le monachisme et la croyance aux miracles, met l'accent à la fin du XVII siècle sur un autre aspect de la doctrine, la morale du chrétien en société ${ }^{12}$. La mise en avant par P.I. de l' «éthique sociale » semble bien se rattacher à cette tendance.

Par ailleurs, d'autres documents décrivant les rapports entre Européens et Russes dans la vie courante révèlent que les « archives de Pskov » de l'anonyme anglais n'étaient pas un cas absolument unique. Il existe d'autres témoignages selon lesquels les étrangers trouvaient chez les Russes compréhension et tolérance, notamment en matière de religion. N.G. Savič, en étudiant les ouvrages étrangers, à la fois dictionnaires et manuels de conversation, des $\mathrm{XVI}^{\mathrm{e}}$ et $\mathrm{XVII}{ }^{\mathrm{e}}$ siècles est parvenue à la conclusion que ces œuvres « témoignent de rapports bienveillants et souvent même amicaux, de contacts variés et approfondis entre étrangers et Russes ; elles montrent l'influence qu'exerçaient l'une sur l'autre les cultures russe et étrangère et qui les enrichissait toutes deux $»^{13}$. Dans l'un de ces ouvrages (la Grammaire russe de Heinrich Wilhelm Ludolf), presque contemporain de la correspondance entre P.I. et R.V. (Ludolf résida à Moscou de 1693 à 1694), de curieux parallèles peuvent être mis en évidence avec les propos tenus par P.I. concernant la religion.

À la fin de son manuel, Ludolf inclut six «dialogues » (en latin, en allemand et en russe) destinés à présenter le parler des « Moscovites ». Le dernier dialogue, intitulé « De l'office divin », rapporte les propos d'un riche citadin russe (probablement un marchand, et selon toute apparence un habitant de Moscou) sur l'Église et la foi. Il déclare notamment :

lorsque je rencontre un homme de bien, je l'aime et le respecte, même si sa foi est différente de la mienne, mais quand je vois un fainéant, je n'éprouve aucun respect pour lui, même s'il est de ma famille. Il est raisonnable de haïr le péché et la méchanceté, mais non pas les hommes. Il est insensé d'en vouloir à quelqu'un parce qu'il n'a pas été élevé de la même façon que nous [...].

12. Paul Bushkovitch, Religion and Society in Russia: the Sixteenth and Seventeenth Centuries, New York : Oxford University Press, 1992, p. 148, 178.

13. Nina Georgievna Savič, «Iz istorii russko-nemeckih kul’turnyh svjazej v XVII v. (nemeckorusskij slovar' razgovornik G. Nevenburg 1629 g.)» [ «Pour une histoire des relations culturelles russo-allemandes au XVII ${ }^{\mathrm{e}}$ siècle (le dictionnaire et manuel de conversation russo-allemand de Heinrich Newenburgk, 1629) »], IZ, 102, 1978, p. 279. Cf. Erika Günther, éd., Das deutschrussische Sprachbuch des Heinrich Newenburgk von 1629. Einführung, sprachliche Analysen, Text, Faksimile, Francfort : Peter Lang, 1999. 
En réponse à son interlocuteur (Ludolf sans doute) qui objecte :

cependant tu vois bien que les Églises se condamnent entre elles,

le Russe rétorque :

En effet, mais nulle part je ne lis que Dieu nous ait donné le pouvoir de nous juger et de nous livrer au diable les uns les autres [...] Et j'ai toujours estimé qu'il était contraire à l'amour chrétien de nous condamner mutuellement, et c'est être orgueilleux en esprit que de considérer tous les autres comme des païens parce qu'ils ne sont pas d'accord avec nous.

De manière plus générale, l'interlocuteur russe décrit le « mode de vie agréable à Dieu » (bogougodnoe žitie) :

nous devons toujours vivre, suivant en cela l'exemple du Sauveur, dans une sage humilité, dans l'amour et la pureté. Tu en trouveras bien peu qui le fassent, presque tous vivent selon la chair, chacun se croit supérieur aux autres, chacun offense autrui et plus de la moitié des hommes ne recherche que les plaisirs de la chair dont ils souillent leur âme.

Selon lui, les véritables chrétiens sont ceux qui s'aiment les uns les autres et non pas ceux qui « estiment qu'ils ont accompli leur devoir de chrétien lorsqu'ils ont prié Dieu et communié à l'heure dite »; il est impossible d'aimer « Dieu ou son prochain », si l'on aime « le monde, c'est-à-dire les richesses, le pouvoir, les honneurs, et les plaisirs de la chair ». Le dialogue s'achève sur cette affirmation :

Que Dieu nous accorde à tous l'esprit de la paix et de vérité qui nous sanctifie et nous éclaire, pour que nous comprenions que tous les vrais chrétiens sont les membres d'un seul et même corps, dont la tête est Jésus-Christ. ${ }^{14}$

Ce sont là des pensées et des dispositions d'esprit très proches de celles exprimées dans la correspondance entre R.V. et P.I. Dans les deux cas, le Russe et l'étranger recherchent dans le domaine de la foi des points de rencontre et les trouvent avant tout dans l'idée que les « chrétiens véritables » (prjamye hristiane) doivent s'aimer les uns les autres, les Russes érigeant l'amour et l'humilité en valeurs primordiales. La confession à laquelle appartient l'individu n'est pas un facteur essentiel, l'important étant d'être « humble » et « homme de bien ». L'opposition entre « spirituel » et « charnel » (ce dernier relevant du péché) est caractéristique de la pensée religieuse de l'habitant de Pskov, comme de l'interlocuteur anonyme de Ludolf.

14. W.H. Ludolf, Russkaja Grammatika [Grammatica russica] Oxford, 1696. Réédition, traduction, préface et commentaires de B.A. Larina, L., 1937, p. 72-92. Cf. Boris Unbegaun, éd., Grammatica Russica : Oxonii A.D. 1696, Oxford : Clarendon Press, 1959. 
Le premier élément de l'opposition se réfère sans équivoque à la foi et à un mode de vie religieux, tandis que le second évoque les « douceurs » du monde profane. «L'éthique du travail » fondée sur la religion revêt une signification toute particulière : «l'oisiveté » (bezdelie) est condamnée précisément en tant que péché.

Les idées religieuses de P.I. trouvent ainsi un écho dans une situation analogue de « rencontre des cultures » et dans un milieu identique, le milieu citadin des marchands. Cela témoigne, nous semble-t-il, d'une tendance particulière de la pensée religieuse russe $\mathrm{au} \mathrm{XVII}^{\mathrm{e}}$ siècle, tendance que confirment d'autres données, hélas fragmentaires, sur les vues de ce milieu en matière d'Église et de foi ${ }^{15}$. Cette tendance est peut-être moins visible que d'autres courants de pensée, qu'il s'agisse de celui, populaire et réactionnaire, des vieux-croyants ou du conservatisme officiel (la politique des derniers patriarches de la fin du XVII ${ }^{\mathrm{e}}$ siècle). Mais si l'on considère l'orientation des réformes de Pierre le Grand, il faut bien reconnaître qu'elle était promise à un bel avenir.

De manière générale, les « archives russes » de R.V. conservées au département des manuscrits de la British Library présentent un intérêt scientifique indéniable, aussi bien dans leur ensemble que dans leurs différentes parties, au premier chef la correspondance privée évoquée ici. L'existence de telles archives est en elle-même un fait remarquable, qui témoigne de l'intérêt que portaient les étrangers (dans le cas présent, les Anglais), à la langue et à la culture russes. La correspondance entre le « répétiteur » de Pskov et son élève constitue un document unique sur les contacts entre représentants de cultures différentes, dans la sphère de la vie quotidienne. Les deux correspondants sont évidemment des gens cultivés, ouverts d'esprit, qui, bien que tournés vers les valeurs traditionnelles, ne s'enferment pas dans leur confession. Ces lettres obligent à reconsidérer les points de vue divergents des historiens, dont certains soutiennent que les étrangers séjournant en Russie au XVII ${ }^{\mathrm{e}}$ siècle ne comprenaient pas, et ne tenaient pas à comprendre, les traditions russes et d'autres, que la société russe avant Pierre le Grand était arriérée et réfractaire aux cultures venant de l'extérieur ${ }^{16}$. Nous constatons que l'étranger intéressé par la culture russe, s'il pouvait se heurter à l'obscurantisme, pouvait tout aussi bien rencontrer des esprits ouverts au dialogue.

Un autre stéréotype concerne la pénétration, dans la Russie du XVII ${ }^{\mathrm{e}}$ siècle, de la civilisation d'Europe occidentale. Dans le cas de P.I. et de R.V., on peut voir

15. Voir par exemple le récit que fait Olearius de ses conversations avec un marchand originaire de Narva : Adam Olearij, Opisanie putešestvija v Moskoviju [Adam Olearius, Description d'un voyage en Moscovie], M., 1996, p. 309-310 (reproduit la trad. de A.M. Lovjagin, M., 1906). Cf. Marshall Poe, éd., Ausfürliche Beschreibung der kundbaren Reyss nach Muscow und Persien, Londres : Routledge Curzon, 2003 (facsim. de l'éd. de Schleswig, 1656) ; Relation du voyage de Moscouie, Tartarie, et de Perse : fait a l'occasion d'vne ambassade, enuoyée au grand-duc de Moscouie, \& au roy de Perse, par le duc de Holstein depuis l'an 1633 iusques en l'an 1639, trad. de l'allemand par Abraham de Wicquefort, P. : Chez Geruais Clouzier, 1656 (nombreuses rééditions).

16. Voir, notamment, Matthew Smith Anderson, Britain's Discovery of Russia, 1553-1815, Londres, New-York : Macmillan, 1958, p. 37, 46-47 ; Inna Lubimenko, Les relations commerciales et politiques de l'Angleterre avec la Russie avant Pierre le Grand, P. : Librairie ancienne Honoré Champion, 1933, p. 275. 
qu'il ne s'agissait pas uniquement d'une « influence européenne » unilatérale, mais d'influences réciproques et d'un dialogue des cultures. Les idées et le mode de vie des Occidentaux constituaient certes un défi à la culture moscovite traditionnelle, mais au niveau de base, celui de la population, la réponse à ce défi ne s'exprimait pas uniquement par l'exacerbation de la spécificité nationale et religieuse, propre aux vieux-croyants notamment. Elle pouvait aussi prendre la forme d'une recherche de valeurs communes culturelles et même religieuses. Comme le montrent les « archives russes » de l'Anglais anonyme, la culture citadine, parce qu'elle était cosmopolite, pouvait être le lieu d'un tel rapprochement.

(traduit du russe par Diane Chertier-Vitovtov)

Institut d'histoire russe, Centre d'histoire de la Russie ancienne Académie des sciences de Russie, Moscou

petr_stefanovich@hotmail.com 